Supplement of Biogeosciences, 12, 5339-5352, 2015

http://www.biogeosciences.net/12/5339/2015/

doi:10.5194/bg-12-5339-2015-supplement

(C) Author(s) 2015. CC Attribution 3.0 License.

(c) (i)

Supplement of

\title{
Responses of leaf traits to climatic gradients: adaptive variation versus compositional shifts
}

\section{T.-T. Meng et al.}

Correspondence to: H. Wang (wanghan_sci@yahoo.com)

The copyright of individual parts of the supplement might differ from the CC-BY 3.0 licence. 


\section{Supplement}

Figure S1: Partial residual plots for the relationships between leaf traits and the CramerPrentice moisture index $(\alpha)$, from a GLM analysis with PFT identity included as a predictor. Each point represents a species-site combination; fitted lines for each PFT are indicated by colours.
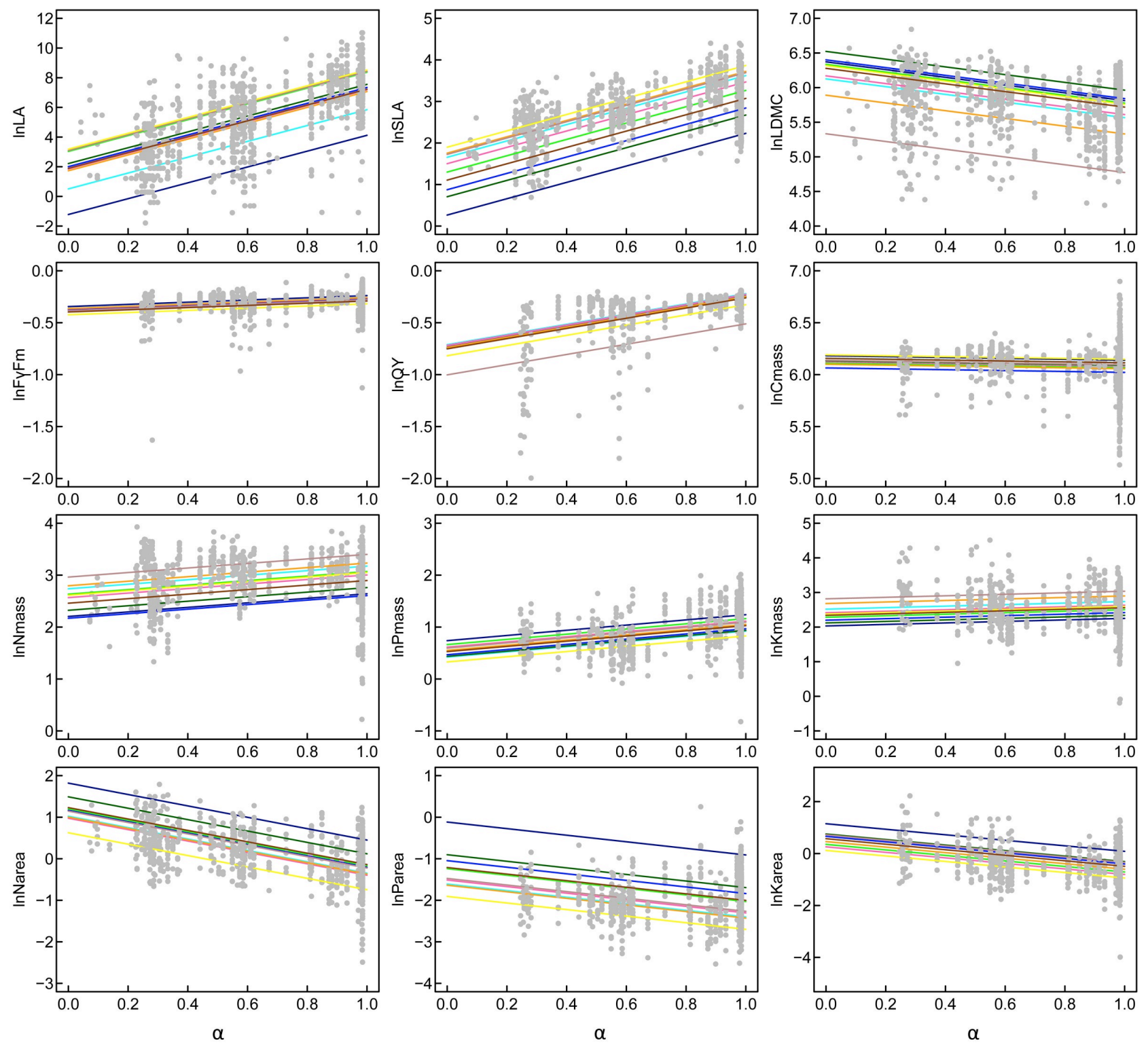

evergreen broadleaf tree

deciduous tree

evergreen shrub

deciduous shrub

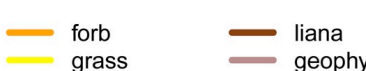

grass geophyte 
Figure S2: Partial residual plots for the relationships between leaf traits and the CramerPrentice moisture index $(\alpha)$, from a GLM analysis with PFT $\times$ climate interactions included as predictors. Each point represents a species-site combination; fitted lines for each PFT are indicated by colours. Only significant PFT $\times$ climate interactions $(\mathrm{P}<0.01)$ are shown.
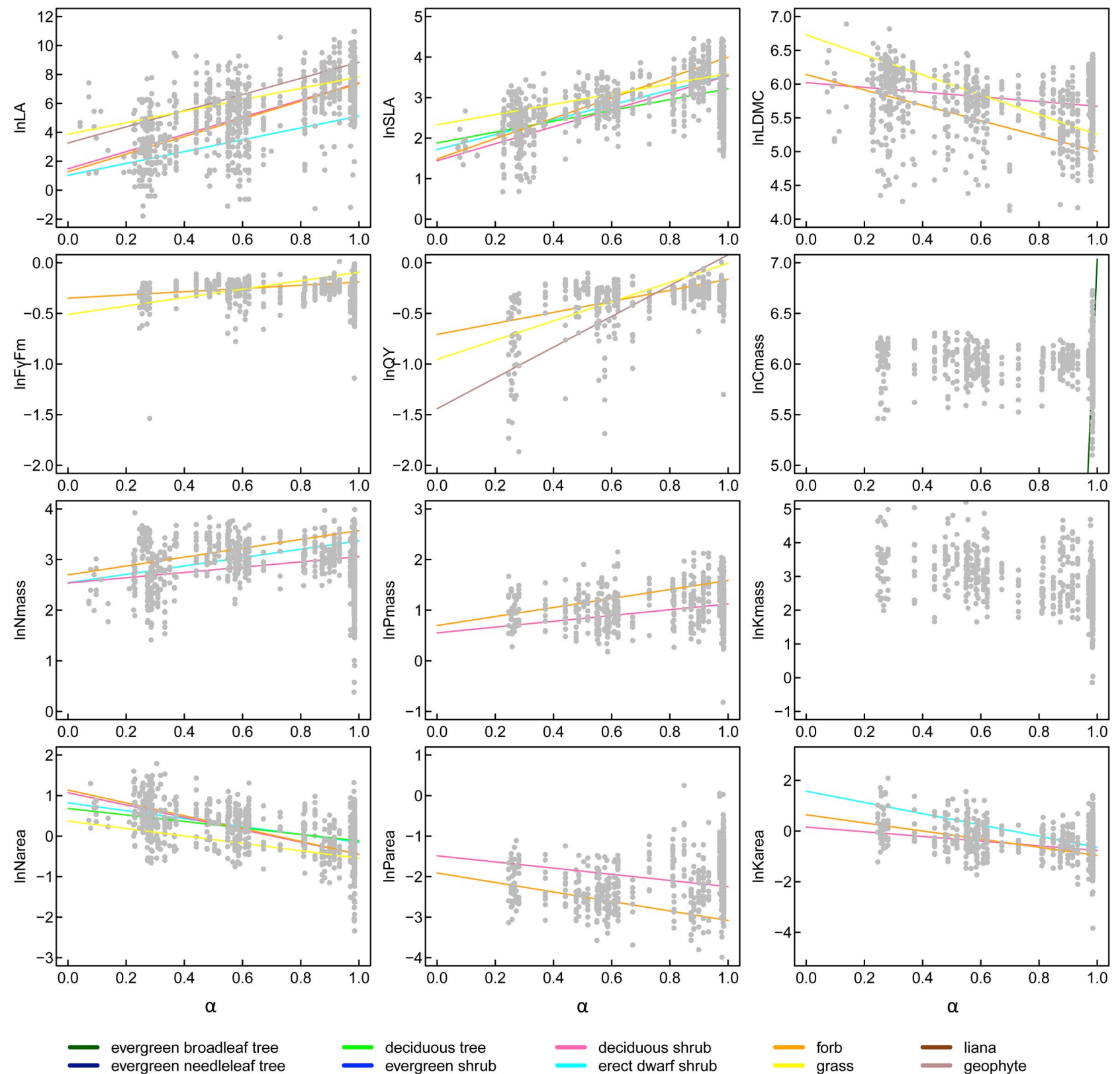

evergreen broadleaf tree
evergreen needleleaf tree

deciduous tree

deciduous shrub

forb

geophyte 
Figure S3: Partial residual plots for the relationships between leaf traits and growing degree days $\left(\mathrm{GDD}_{0}\right)$, from a GLM analysis with PFT identity included as a predictor. Each point represents a species-site combination; fitted lines for each PFT are indicated by colours.
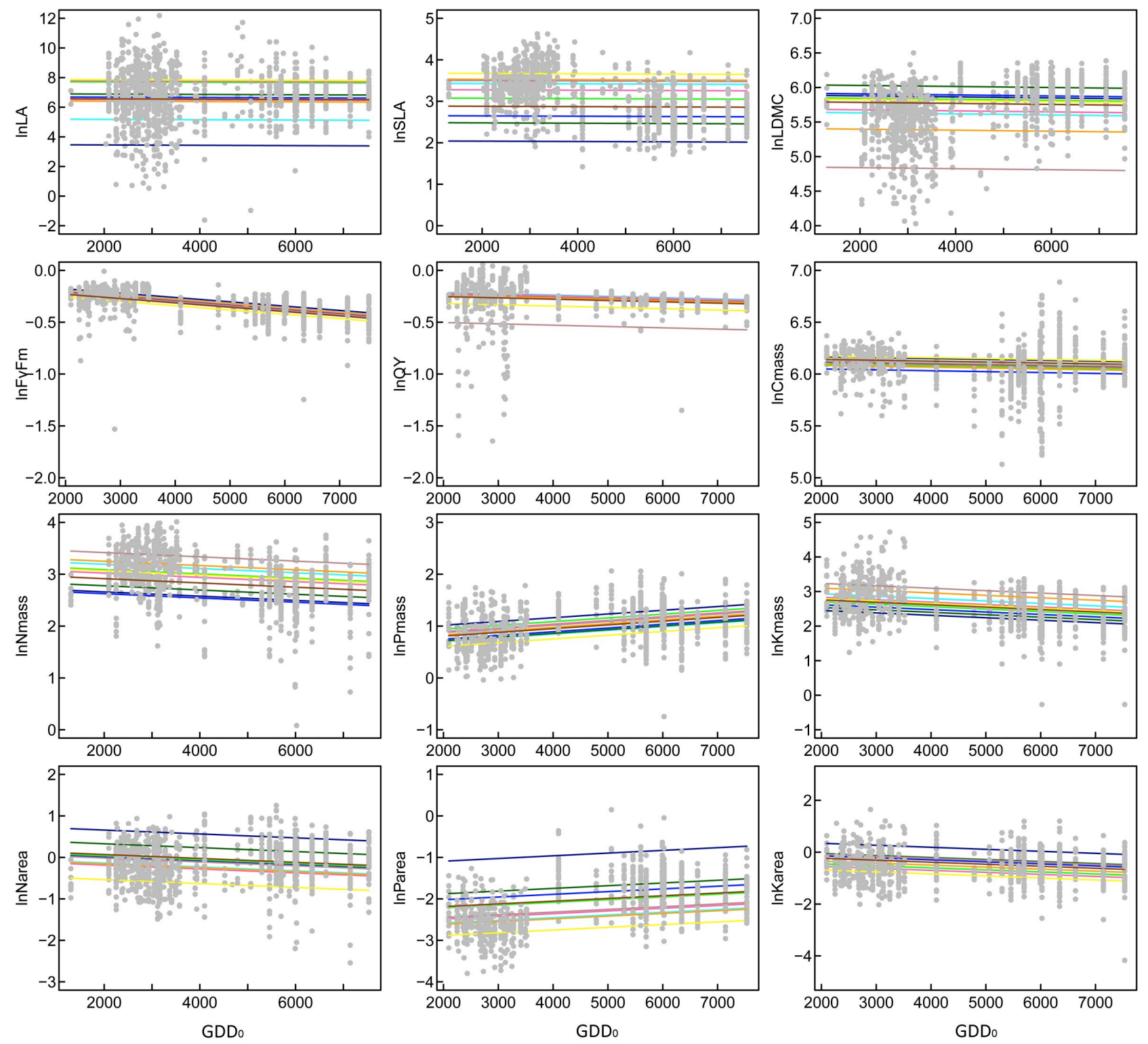
Figure S4: Partial residual plots for the relationships between leaf traits and growing degree days $\left(\mathrm{GDD}_{0}\right)$, from a GLM analysis with PFT $\times$ climate interactions included as predictors. Each point represents a species-site combination; fitted lines for each PFT are indicated by colours. Only significant $\mathrm{PFT} \times$ climate interactions $(\mathrm{P}<0.01)$ are shown.
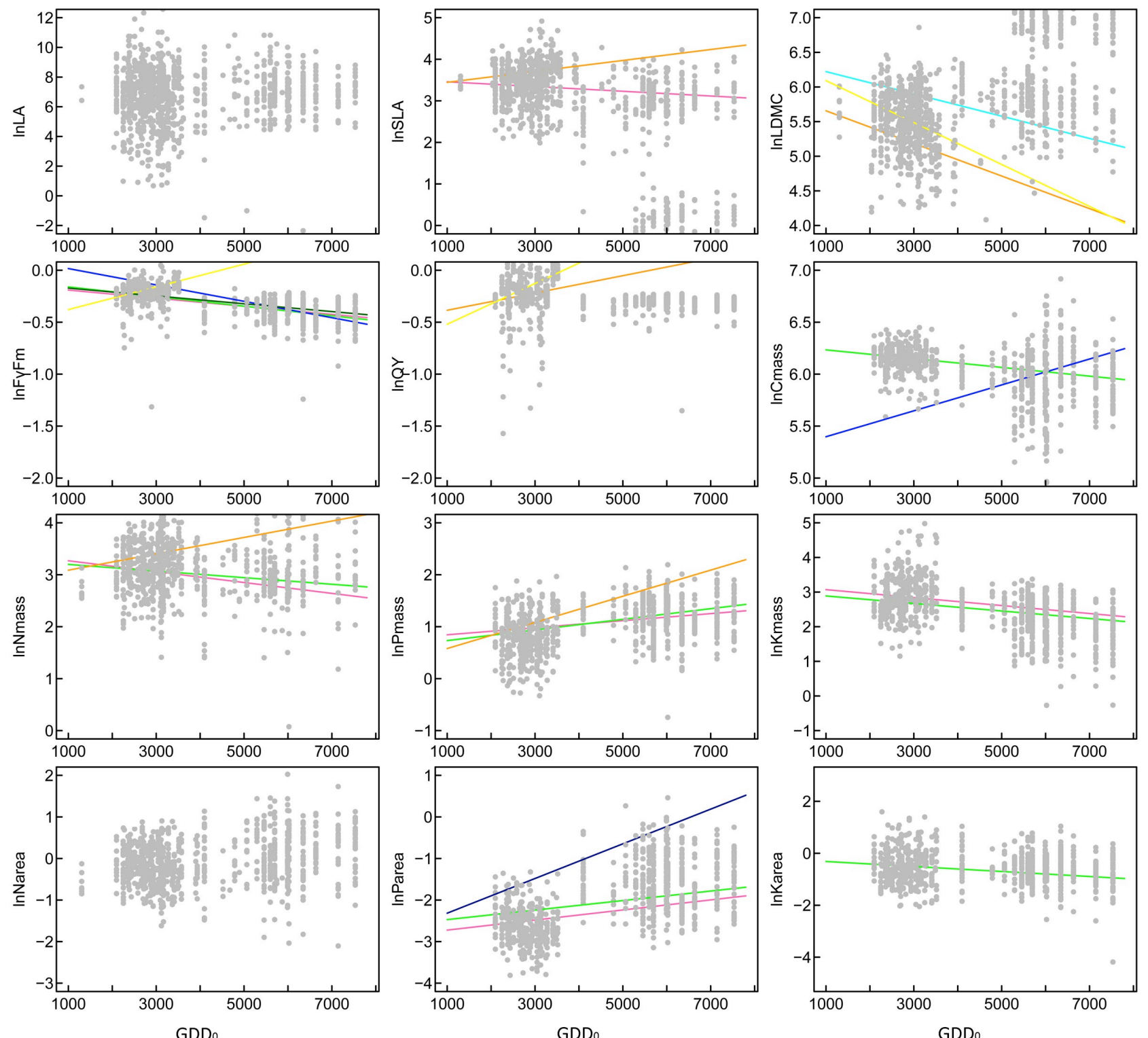
Table S1: Characteristics of sampling sites

\begin{tabular}{|c|c|c|c|c|c|c|c|}
\hline site code & $\begin{array}{l}\text { latitude } \\
\left({ }^{\circ} \mathrm{N}\right)\end{array}$ & $\begin{array}{l}\text { longitude } \\
\left({ }^{\circ} \mathrm{E}\right)\end{array}$ & $\begin{array}{l}\text { elevation } \\
(\mathrm{m})\end{array}$ & vegetation type & $\begin{array}{l}\text { number } \\
\text { of } \\
\text { species }\end{array}$ & $\begin{array}{l}\mathrm{GDD}_{0} \\
\left(\text { day }^{\circ} \mathrm{C}\right)\end{array}$ & $\alpha$ \\
\hline NECT01 & 42.88 & 118.48 & 1024 & steppe & 19 & 2433.20 & 0.67 \\
\hline NECT02 & 43.64 & 119.02 & 781 & steppe & 43 & 3047.57 & 0.55 \\
\hline NECT03 & 43.02 & 129.78 & 136 & $\begin{array}{l}\text { deciduous broad- } \\
\text { leaved forest }\end{array}$ & 24 & 2727.77 & 0.90 \\
\hline NECT04 & 42.98 & 130.08 & 114 & $\begin{array}{l}\text { evergreen } \\
\text { conifer/deciduous } \\
\text { broad-leaved } \\
\text { forest }\end{array}$ & 26 & 2870.71 & 0.88 \\
\hline NECT05 & 43.30 & 131.15 & 289 & $\begin{array}{l}\text { deciduous } \\
\text { conifer/deciduous } \\
\text { broad-leaved } \\
\text { forest }\end{array}$ & 42 & 2391.45 & 0.92 \\
\hline NECT06 & 43.12 & 131.00 & 244 & $\begin{array}{l}\text { evergreen } \\
\text { conifer/deciduous } \\
\text { broad-leaved } \\
\text { forest }\end{array}$ & 49 & 2097.04 & 0.98 \\
\hline NECT07 & 43.39 & 129.67 & 224 & $\begin{array}{l}\text { deciduous } \\
\text { conifer/deciduous } \\
\text { broad-leaved } \\
\text { forest }\end{array}$ & 40 & 2760.26 & 0.87 \\
\hline NECT08 & 43.25 & 128.64 & 601 & $\begin{array}{l}\text { evergreen } \\
\text { conifer/deciduous } \\
\text { broad-leaved } \\
\text { forest }\end{array}$ & 39 & 2702.15 & 0.93 \\
\hline NECT09 & 43.73 & 127.03 & 390 & $\begin{array}{l}\text { evergreen } \\
\text { conifer/deciduous } \\
\text { broad-leaved } \\
\text { forest }\end{array}$ & 55 & 2935.09 & 0.98 \\
\hline NECT10 & 43.81 & 125.68 & 252 & $\begin{array}{l}\text { evergreen } \\
\text { conifer/deciduous } \\
\text { broad-leaved } \\
\text { forest }\end{array}$ & 40 & 3278.10 & 0.81 \\
\hline NECT11 & 44.59 & 123.51 & 146 & meadow steppe & 19 & 3412.10 & 0.58 \\
\hline NECT12 & 44.43 & 123.27 & 150 & meadow steppe & 18 & 3407.62 & 0.57 \\
\hline NECT13 & 43.60 & 121.84 & 203 & meadow steppe & 20 & 3515.92 & 0.49 \\
\hline NECT14 & 44.12 & 121.77 & 202 & meadow steppe & 7 & 3490.75 & 0.50 \\
\hline NECT15 & 44.39 & 120.55 & 448 & steppe & 21 & 3047.41 & 0.59 \\
\hline NECT16 & 44.22 & 120.37 & 372 & steppe & 18 & 3248.01 & 0.55 \\
\hline NECT17 & 43.88 & 119.38 & 601 & steppe & 15 & 2683.63 & 0.62 \\
\hline NECT18 & 43.76 & 119.12 & 729 & steppe & 23 & 2816.77 & 0.59 \\
\hline NECT19 & 43.34 & 118.49 & 707 & steppe & 12 & 2646.25 & 0.61 \\
\hline NECT20 & 43.19 & 117.76 & 889 & steppe & 23 & 2583.25 & 0.62 \\
\hline NECT21 & 43.22 & 117.24 & 1259 & steppe & 23 & 2248.48 & 0.61 \\
\hline NECT22 & 43.39 & 116.89 & 1267 & steppe & 13 & 2274.88 & 0.58 \\
\hline
\end{tabular}




\begin{tabular}{|c|c|c|c|c|c|c|c|}
\hline NECT23 & 43.55 & 116.68 & 1261 & steppe & 22 & 2410.95 & 0.56 \\
\hline NECT24 & 43.69 & 116.64 & 1211 & steppe & 20 & 2374.09 & 0.52 \\
\hline NECT25 & 43.91 & 116.31 & 1199 & steppe & 24 & 2666.32 & 0.48 \\
\hline NECT26 & 43.90 & 115.32 & 1196 & steppe & 27 & 2509.65 & 0.44 \\
\hline NECT27 & 43.94 & 114.61 & 1123 & desert steppe & 19 & 2716.00 & 0.37 \\
\hline NECT28 & 43.83 & 113.83 & 1166 & desert steppe & 14 & 2895.45 & 0.28 \\
\hline NECT29 & 43.80 & 113.36 & 1017 & desert steppe & 11 & 3104.24 & 0.25 \\
\hline NECT30 & 43.72 & 112.59 & 974 & desert steppe & 21 & 3103.83 & 0.27 \\
\hline NECT31 & 43.63 & 112.17 & 999 & desert steppe & 16 & 3164.51 & 0.26 \\
\hline NECT32 & 43.66 & 111.92 & 1005 & desert steppe & 15 & 3127.61 & 0.26 \\
\hline NECT33 & 43.65 & 111.89 & 1017 & desert steppe & 16 & 3129.06 & 0.27 \\
\hline NSTEC01 & 36.24 & 117.02 & 368 & $\begin{array}{l}\text { conifer/deciduous } \\
\text { broad-leaved } \\
\text { forest }\end{array}$ & 9 & 4787.79 & 0.73 \\
\hline NSTEC02 & 34.64 & 119.24 & 59 & $\begin{array}{l}\text { conifer/deciduous } \\
\text { broad-leaved } \\
\text { forest }\end{array}$ & 13 & 5063.76 & 0.85 \\
\hline NSTEC03 & 32.05 & 118.86 & 76 & $\begin{array}{l}\text { conifer/deciduous } \\
\text { broad-leaved } \\
\text { forest }\end{array}$ & 35 & 5598.22 & 0.98 \\
\hline NSTEC04 & 30.29 & 119.44 & 299 & $\begin{array}{l}\text { evergreen broad- } \\
\text { leaved forest }\end{array}$ & 21 & 5294.63 & 0.98 \\
\hline NSTEC05 & 29.80 & 121.79 & 231 & $\begin{array}{l}\text { evergreen broad- } \\
\text { leaved forest }\end{array}$ & 41 & 6022.80 & 0.98 \\
\hline NSTEC06 & 27.98 & 119.14 & 294 & $\begin{array}{l}\text { evergreen broad- } \\
\text { leaved forest }\end{array}$ & 57 & 5992.46 & 0.98 \\
\hline NSTEC07 & 26.59 & 118.05 & 239 & $\begin{array}{l}\text { evergreen broad- } \\
\text { leaved forest }\end{array}$ & 59 & 6345.35 & 0.98 \\
\hline NSTEC08 & 24.41 & 116.34 & 195 & $\begin{array}{l}\text { evergreen broad- } \\
\text { leaved forest }\end{array}$ & 35 & 7143.63 & 0.99 \\
\hline NSTEC09 & 23.17 & 112.54 & 240 & $\begin{array}{l}\text { evergreen broad- } \\
\text { leaved forest }\end{array}$ & 45 & 7532.60 & 0.99 \\
\hline NSTEC10 & 25.32 & 110.25 & 199 & $\begin{array}{l}\text { conifer/deciduous } \\
\text { broad-leaved } \\
\text { forest }\end{array}$ & 29 & 6635.23 & 0.99 \\
\hline NSTEC11 & 26.84 & 109.60 & 390 & $\begin{array}{l}\text { conifer/deciduous } \\
\text { broad-leaved } \\
\text { forest }\end{array}$ & 53 & 5697.20 & 0.99 \\
\hline NSTEC12 & 28.34 & 109.73 & 220 & $\begin{array}{l}\text { conifer/deciduous } \\
\text { broad-leaved } \\
\text { forest }\end{array}$ & 39 & 5457.14 & 0.99 \\
\hline NSTEC13 & 33.50 & 111.49 & 449 & $\begin{array}{l}\text { deciduous broad- } \\
\text { leaved forest }\end{array}$ & 27 & 4098.36 & 0.97 \\
\hline NSTEC14 & 39.95 & 115.42 & 1253 & $\begin{array}{l}\text { deciduous broad- } \\
\text { leaved forest }\end{array}$ & 14 & 2357.31 & 0.81 \\
\hline X01 & 48.19 & 87.02 & 272 & desert & 8 & 2252.67 & 0.33 \\
\hline X02 & 46.40 & 85.95 & 701 & desert & 18 & 3575.09 & 0.23 \\
\hline
\end{tabular}




\begin{tabular}{|c|c|c|c|c|c|c|c|}
\hline X03 & 47.04 & 87.09 & 620 & desert steppe & 10 & 2930.53 & 0.31 \\
\hline X04 & 47.83 & 86.85 & 499 & desert steppe & 20 & 3118.13 & 0.29 \\
\hline X05 & 47.94 & 86.83 & 481 & desert & 11 & 3105.78 & 0.29 \\
\hline X06 & 48.17 & 87.08 & 709 & desert steppe & 15 & 2252.67 & 0.33 \\
\hline X07 & 48.11 & 87.01 & 1100 & shrubland & 6 & 2252.67 & 0.33 \\
\hline X08 & 48.33 & 87.12 & 1595 & meadow & 13 & 1304.34 & 0.57 \\
\hline X09 & 47.72 & 87.02 & 498 & desert steppe & 23 & 3165.82 & 0.28 \\
\hline $\mathrm{X} 10$ & 47.74 & 87.54 & 521 & desert steppe & 13 & 3146.09 & 0.27 \\
\hline X11 & 47.16 & 88.70 & 750 & desert & 8 & 3159.38 & 0.3 \\
\hline $\mathrm{X} 12$ & 46.30 & 89.55 & 885 & desert & 10 & 3021.81 & 0.32 \\
\hline X13 & 45.36 & 89.40 & 1068 & desert & 7 & 2929.95 & 0.33 \\
\hline X14 & 44.12 & 87.81 & 513 & desert & 11 & 3901.31 & 0.29 \\
\hline X15 & 44.08 & 87.79 & 583 & desert steppe & 18 & 3934.76 & 0.29 \\
\hline X16 & 44.07 & 88.08 & 852 & desert steppe & 11 & 3367.67 & 0.35 \\
\hline $\mathrm{X} 17$ & 44.00 & 88.06 & 1060 & meadow & 12 & 3369.92 & 0.31 \\
\hline X18 & 43.93 & 88.11 & 1430 & shrubland & 9 & 3153.82 & 0.37 \\
\hline X19 & 42.84 & 89.44 & -91 & shrubland & 2 & 5745.44 & 0.09 \\
\hline X20 & 42.73 & 89.44 & -136 & desert & 2 & 5869.71 & 0.14 \\
\hline X21 & 42.69 & 89.42 & -146 & desert & 2 & 5989.24 & 0.08 \\
\hline X22 & 42.37 & 88.57 & 1721 & desert & 5 & 3112.29 & 0.28 \\
\hline X23 & 42.22 & 87.76 & 1445 & desert & 9 & 3093.93 & 0.23 \\
\hline X24 & 41.81 & 86.25 & 1444 & desert & 3 & 3617.6 & 0.19 \\
\hline X26 & 40.83 & 84.29 & 921 & desert & 4 & 4893.58 & 0.07 \\
\hline X27 & 41.48 & 84.21 & 928 & desert & 3 & 4678.9 & 0.21 \\
\hline X28 & 41.50 & 84.51 & 919 & desert & 3 & 4644.14 & 0.11 \\
\hline X29 & 41.66 & 84.89 & 902 & desert & 5 & 4520.84 & 0.10 \\
\hline X30 & 40.51 & 89.11 & 70 & desert & 3 & 4865.42 & 0.04 \\
\hline X31 & 48.33 & 87.12 & 1595 & desert & 1 & 1304.34 & 0.57 \\
\hline X32 & 40.83 & 84.29 & 26 & desert & 1 & 4893.58 & 0.07 \\
\hline X33 & 43.93 & 88.11 & 1430 & shrubland & 3 & 3153.82 & 0.37 \\
\hline X34 & 43.90 & 88.12 & 1935 & $\begin{array}{l}\text { evergreen conifer } \\
\text { forest }\end{array}$ & 9 & 2037.89 & 0.70 \\
\hline
\end{tabular}


Table S2. Species sampled at each site.

\begin{tabular}{|c|c|}
\hline Species & Sampling sites \\
\hline Abelia biflora & NSTEC14 \\
\hline Acanthopanax senticosus & NECT05, NECT06, NECT09 \\
\hline Acanthopanax trifoliatus & NSTEC12 \\
\hline Acer buergerianum & NSTEC03, NSTEC13 \\
\hline Acer cinnamomifolium & NSTEC11 \\
\hline Acer cordatum & NSTEC06 \\
\hline Acer ginnala & NECT05, NECT06 \\
\hline Acer mandschuricum & NECT09 \\
\hline Acer mono & NECT03, NECT06, NECT08, NECT09, NSTEC14 \\
\hline Acer tegmentosum & NECT09 \\
\hline Achnatherum sibiricum & NECT23, NECT26 \\
\hline Acmena acuminatissima & NSTEC09 \\
\hline $\begin{array}{l}\text { Aconitum jaluense var. } \\
\text { paniculigerum }\end{array}$ & NECT09 \\
\hline Aconitum kirinense & NECT08 \\
\hline Aconitum volubile & NECT06 \\
\hline Acronychia pedunculata & NSTEC09 \\
\hline Actaea asiatica & NECT08 \\
\hline Actinidia arguta & NECT09 \\
\hline Actinidia eriantha & NSTEC07 \\
\hline Adenophora gmelinii & NECT25 \\
\hline $\begin{array}{l}\text { Adenophora stenanthina var. } \\
\text { crispata }\end{array}$ & NECT25 \\
\hline Adenophora tetraphylla & NECT05, NECT06, NECT07 \\
\hline Adiantum pedatum & NECT09 \\
\hline Adina pilulifera & NSTEC06, NSTEC07 \\
\hline Adinandra megaphylla & NSTEC06 \\
\hline Aegopodium alpestre & NECT05, NECT06, NECT07, NECT09 \\
\hline Agrimonia pilosa & NECT05, NECT07 \\
\hline Agropyron cristatum & NECT02 \\
\hline Agropyron michnoi & $\begin{array}{l}\text { NECT17, NECT19, NECT20, NECT21, NECT23, NECT24, NECT25, NECT26, } \\
\text { NECT27, NECT28, NECT29, NECT31, NECT33 }\end{array}$ \\
\hline Ajania achilloides & NECT28 \\
\hline Akebia trifoliata & NSTEC12 \\
\hline Alangium chinense & NSTEC03 \\
\hline Albizia kalkora & NSTEC02, NSTEC10, NSTEC13 \\
\hline Alchornea trewioides & NSTEC10 \\
\hline Allium bidentatum & NECT29 \\
\hline Allium condensatum & NECT23, NECT26 \\
\hline Allium leucocephalum & NECT29 \\
\hline Allium mongolicum & $\begin{array}{l}\text { NECT15, NECT18, NECT21, NECT23, NECT24, NECT25, NECT26, NECT27, } \\
\text { NECT28 }\end{array}$ \\
\hline Allium neriniflorum & NECT20 \\
\hline Allium polyrhizum & NECT27 \\
\hline Allium ramosum & $\begin{array}{l}\text { NECT10, NECT17, NECT22, NECT23, NECT24, NECT25, NECT26, NECT27, } \\
\text { NECT28, NECT30, NECT31, NECT32, NECT33 }\end{array}$ \\
\hline Allium senescens & NECT01, NECT11 \\
\hline Allium sp. & NECT02, NECT13 \\
\hline Allium tenuissimum & NECT32 \\
\hline Alyxia vulgaris & NSTEC07, NSTEC08 \\
\hline Amaranthus retroflexus & NECT12 \\
\hline Ampelopsis cantoniensis & NSTEC06 \\
\hline
\end{tabular}




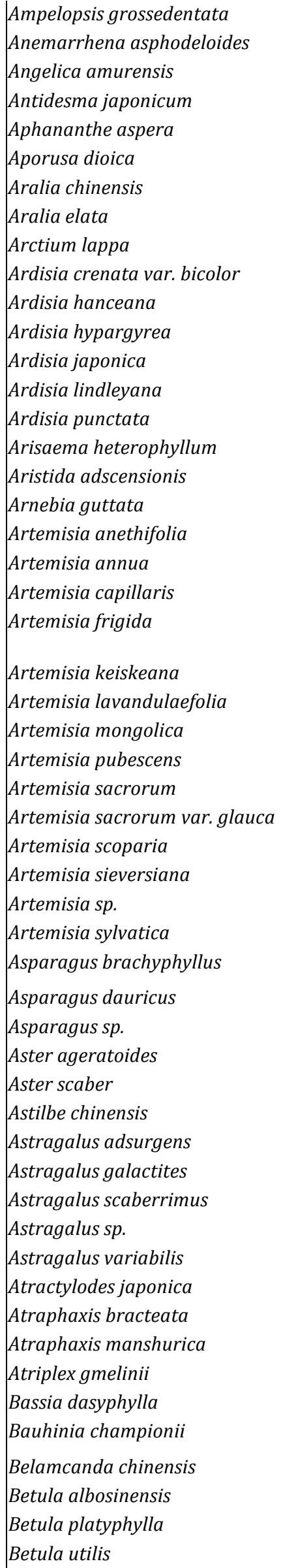

NSTEC07

NECT02

NECT06

NSTEC06, NSTEC07, NSTEC12

NSTEC03

NSTEC09

NSTEC12

NECT09

NECT06, NECT08

NSTEC05

NSTEC09

NSTEC09

NSTEC05

NSTEC07, NSTEC08

NSTEC09

NECT09

NECT14, NECT16, NECT30

NECT18, NECT20

NECT11

NECT17, NECT18

NSTEC13

NECT01, NECT02, NECT15, NECT18, NECT20, NECT21, NECT22, NECT23, NECT24, NECT25, NECT26, NECT27, NECT28, NECT30

NECT07

NECT10

NECT10, NECT17

NECT27

NECT01, NECT02, NECT10, NECT15

NECT01, NECT02, NECT15

NECT02, NECT10, NECT11, NECT12, NECT14, NECT20, NECT21, NECT22

NECT10

NECT02, NECT04, NECT32

NECT04

NSTEC13

NECT04, NECT27, NECT28, NECT29, NECT30, NECT31, NECT32, NECT33

NECT05, NECT08, NECT09

NECT11

NECT06, NECT07, NECT08

NECT03, NECT05, NECT06, NECT07, NECT08, NECT09

NECT12, NECT21, NECT26

NECT01, NECT02, NECT26

NECT01, NECT02, NECT26

NECT30

NECT25

NECT07

NECT30

NECT18

NECT13

NECT19

NSTEC10

NECT18

NECT05

NECT05, NECT06

NECT03, NECT07 


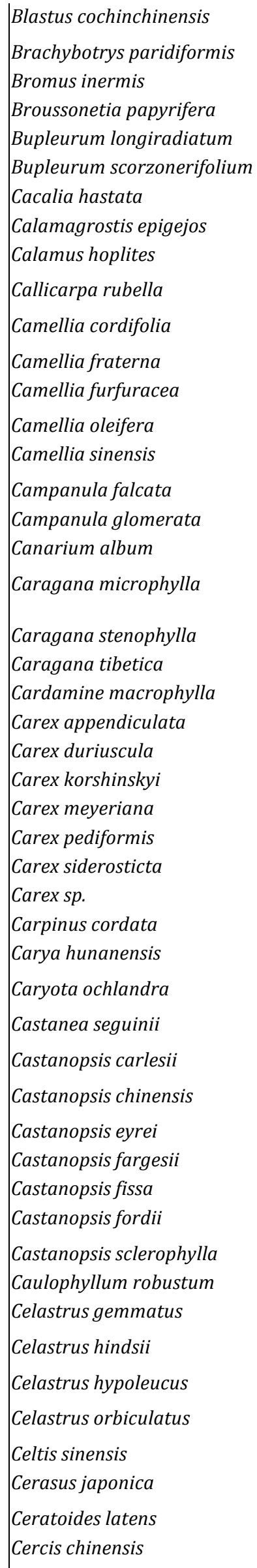

NSTEC09

NECT06, NECT09

NECT05

NSTEC01

NECT07

NECT23, NECT25, NECT26

NECT05, NECT06, NECT08, NECT09

NECT04, NECT11

NSTEC09

NSTEC06

NSTEC07

NSTEC05, NSTEC06, NSTEC07

NSTEC11

NSTEC05, NSTEC11, NSTEC12

NSTEC06

NECT10

NECT05

NSTEC09

NECT02, NECT16, NECT19, NECT23, NECT24, NECT25, NECT27, NECT28, NECT29, NECT30, NECT31, NECT33

NECT26, NECT27, NECT28, NECT29, NECT30, NECT31, NECT33

NECT30

NECT09, NECT10

NECT06

NECT10, NECT12, NECT22, NECT30

NECT21

NECT09

NECT03, NECT05, NECT06

NECT08, NECT09

NECT04, NECT05, NECT07, NECT09

NECT09

NSTEC11

NSTEC09

NSTEC12

NSTEC05

NSTEC09

NSTEC04, NSTEC06, NSTEC07

NSTEC05, NSTEC07, NSTEC08, NSTEC12

NSTEC06, NSTEC07, NSTEC08, NSTEC09

NSTEC07

NSTEC04, NSTEC05, NSTEC06

NECT06, NECT07

NSTEC11

NSTEC10

NSTEC07

NSTEC05

NSTEC03, NSTEC10, NSTEC11, NSTEC12

NSTEC02

NECT32, NECT33

NSTEC03 
Chenopodium acuminatum

Chenopodium album

Chenopodium aristatum

Chenopodium glaucum

Chloranthus japonicus

Chloris virgata

Choerospondias axillaris

Chrysosplenium alternifolium

Cinnamomum appelianum

Cinnamomum camphora

Cirsium setosum

Cleistogenes songorica

Cleistogenes squarrosa

Clematis armandii

Clematis fusca var violaceae

Clematis hexapetala

Clematis sp.

Clematis terniflora var. mandshurica

Clerodendrum mandarinorum

Clerodendrum trichotomum

Cleyera japonica

Clintonia udensis

Convallaria majalis

Convolvulus ammannii

Convolvulus arvensis

Coptosapelta diffusa

Coriaria nepalensis

Corylus heterophylla

Cotinus coggygria

Crataegus pinnatifida

Crataegus sp.

Croton tiglium

Cryptocarya chinensis

Cryptocarya concinna

Cryptomeria fortunei

Cudrania cochinchinensis

Cudrania tricuspidata

Cunninghamia lanceolata

Cuscuta chinensis

Cyclobalanopsis gilva

Cyclobalanopsis glauca

Cyclobalanopsis gracilis

Cyclobalanopsis stewardiana

Cymbaria dahurica

Cynanchum sibiricum

Cynanchum thesioides

Dalbergia hancei

Dalbergia hupeana

Dalbergia mimosoides
NECT14, NECT16, NECT19, NECT27

NECT23, NECT24

NECT16, NECT20, NECT24

NECT13

NECT06, NECT08

NECT11, NECT12, NECT13, NECT19, NECT25

NSTEC07

NECT06

NSTEC12

NSTEC03, NSTEC06

NECT24

NECT28, NECT30, NECT31, NECT33

NECT01, NECT02, NECT15, NECT16, NECT17, NECT18, NECT20, NECT21, NECT22, NECT24

NSTEC11, NSTEC13

NECT07

NECT10

NECT03, NECT04, NECT06, NECT09

NECT10

NSTEC12

NSTEC02

NSTEC05

NECT08

NECT07

NECT27, NECT28, NECT30, NECT31, NECT32, NECT33

NECT15, NECT20, NECT21

NSTEC06, NSTEC07, NSTEC08

NSTEC13

NECT03, NECT05, NECT06, NECT07, NECT08, NECT09, NECT10, NSTEC14

NSTEC13

NECT10

NECT05

NSTEC10

NSTEC09

NSTEC09

NSTEC06

NSTEC10

NSTEC03

NSTEC04, NSTEC06, NSTEC12

NECT16

NSTEC05

NSTEC04, NSTEC05, NSTEC06, NSTEC07, NSTEC10, NSTEC11, NSTEC12 NSTEC05

NSTEC05

NECT23, NECT26, NECT27

NECT18, NECT19

NECT02

NSTEC11

NSTEC02, NSTEC04, NSTEC10, NSTEC13

NSTEC05 


\begin{tabular}{|c|c|}
\hline Damnacanthus indicus & NSTEC05 \\
\hline Daphniphyllum oldhamii & NSTEC07, NSTEC08, NSTEC11 \\
\hline Dasymaschalon rostratum & NSTEC09 \\
\hline Decaspermum fruticosum & NSTEC10 \\
\hline Delphinium grandiflorum & NECT02, NECT18 \\
\hline Dendrotrophe frutescens & NSTEC08 \\
\hline $\begin{array}{l}\text { Desmodium fallax var. } \\
\text { mandshuricum }\end{array}$ & NECT09 \\
\hline Dianthus chinensis & NECT18, NECT20, NECT21 \\
\hline Dichroa febrifuga & NSTEC11 \\
\hline Dioscorea cirrhosa & NSTEC05 \\
\hline Dioscorea nipponica & NECT03, NECT04, NECT05, NECT06, NECT09 \\
\hline Dioscorea opposita & NSTEC05 \\
\hline Diospyros cathayensis & NSTEC12 \\
\hline Diospyros eriantha & NSTEC09 \\
\hline Diospyros kaki var. sylvestris & NSTEC07, NSTEC12 \\
\hline Diospyros lotus & NSTEC04, NSTEC13 \\
\hline Diospyros miaoshanica & NSTEC11 \\
\hline Diospyros morrisiana & NSTEC08 \\
\hline Diospyros tutcheri & NSTEC08 \\
\hline Diploclisia glaucescens & NSTEC07 \\
\hline Dischidia chinensis & NSTEC09 \\
\hline Distylium myricoides & NSTEC06 \\
\hline Dontostemon integrifolius & NECT20, NECT21 \\
\hline Dracocephalum argunense & NECT07 \\
\hline Dryopteris crassirhizoma & NECT09 \\
\hline Echinochloa crusgalli & NECT10, NECT11, NECT12 \\
\hline Echinops gmelinii & NECT16, NECT19, NECT24 \\
\hline Echinops sp. & NECT02 \\
\hline Elaeagnus henryi & NSTEC12 \\
\hline Elaeocarpus glabripetalus & NSTEC08 \\
\hline Elaeocarpus japonicus & NSTEC05, NSTEC06, NSTEC07 \\
\hline Embelia rudis & NSTEC06, NSTEC07 \\
\hline Engelhardtia roxburghiana & NSTEC08 \\
\hline Ephedra distachya & NECT17 \\
\hline Ephedra sinica & NECT26 \\
\hline Equisetum arvense & NECT06 \\
\hline Equisetum hyemale & NECT05, NECT06 \\
\hline Eragrostis cilianensis & NECT16 \\
\hline Eragrostis minor & NECT12, NECT14, NECT30, NECT31 \\
\hline Erodium stephanianum & NECT02, NECT13 \\
\hline Erycibe obtusifolia & NSTEC09 \\
\hline Erythrophleum fordii & NSTEC09 \\
\hline Euonymus alatus & NECT03, NECT05, NECT06, NECT08, NECT09, NSTEC03 \\
\hline Euonymus dielsianus & NSTEC11 \\
\hline Euonymus pauciflorus & NECT07, NECT09 \\
\hline Euphorbia esula & NECT01, NECT02, NECT20 \\
\hline Euphorbia humifusa & NECT01, NECT02, NECT31 \\
\hline Euphorbia humifusa var. pilosa & NECT16 \\
\hline Euphorbia lucorum & NECT07 \\
\hline
\end{tabular}




\begin{tabular}{|c|c|}
\hline Euphorbia mandshurica & NECT10 \\
\hline Eurya alata & NSTEC12 \\
\hline Eurya loquaiana & NSTEC11 \\
\hline Eurya nitida & NSTEC07 \\
\hline Eurya rubiginosa & NSTEC05 \\
\hline Eurya rubiginosa var. attenuata & NSTEC04 \\
\hline Euscaphis japonica & NSTEC06 \\
\hline Evodia fargesii & NSTEC08 \\
\hline Evodia lepta & NSTEC08 \\
\hline Ferula bungeana & NECT16, NECT30, NECT31, NECT33 \\
\hline Festuca dahurica & NECT29 \\
\hline Ficus fulva & NSTEC07, NSTEC08 \\
\hline Ficus henryi & NSTEC11 \\
\hline Ficus heteromorpha & NSTEC12 \\
\hline Ficus pandurata & NSTEC06 \\
\hline Ficus pandurata var. angustifolia & NSTEC12 \\
\hline Ficus pumila & NSTEC06 \\
\hline Ficus sarmentosa var. henryi & NSTEC12 \\
\hline Ficus variolosa & NSTEC10 \\
\hline Ficus wightiana & NSTEC09 \\
\hline Filifolium sibiricum & NECT21 \\
\hline Firmiana platanifolia & NSTEC03, NSTEC10 \\
\hline Fissistigma glaucescens & NSTEC09 \\
\hline Fissistigma oldhamii & NSTEC07 \\
\hline Fordia cauliflora & NSTEC10 \\
\hline Fragaria orientalis & NECT08, NECT09 \\
\hline Fraxinus bungeana & NSTEC14 \\
\hline Fraxinus mandshurica & NECT05, NECT06 \\
\hline Fraxinus rhynchophylla & NECT03, NECT04, NECT05, NECT06, NECT08 \\
\hline Galium manshuricum & NECT08 \\
\hline Galium verum & NECT23 \\
\hline Garcinia oblongifolia & NSTEC09 \\
\hline Gardenia jasminoides & NSTEC04, NSTEC07, NSTEC08, NSTEC11 \\
\hline Gentiana dahurica & NECT25 \\
\hline Geranium sp. & NECT08 \\
\hline Geranium transbaicalicum & NECT18, NECT20 \\
\hline Geum sp. & NECT05 \\
\hline Gironniera subaequalis & NSTEC09 \\
\hline Glochidion eriocarpum & NSTEC08 \\
\hline Glochidion puberum & NSTEC02, NSTEC03 \\
\hline Glycine soja & NECT09 \\
\hline Glycyrrhiza uralensis & NECT02, NECT23, NECT26 \\
\hline Gnetum montanum & NSTEC09 \\
\hline Gnetum parvifolium & NSTEC07 \\
\hline Grewia biloba & NSTEC01, NSTEC10 \\
\hline Haplophyllum dauricum & NECT02, NECT26, NECT30, NECT31, NECT32 \\
\hline Haplophyllum tragacanthoides & NECT32 \\
\hline Hedysarum fruticosum & NECT15, NECT17 \\
\hline
\end{tabular}




\begin{tabular}{|c|}
\hline $\mid \begin{array}{l}\text { Helicia cochinchinensis } \\
\text { Hemerocallis middendorffii } \\
\text { Heteropappus altaicus }\end{array}$ \\
\hline Hieracium denticulata \\
\hline Hieracium denticuliferum \\
\hline Hippolytia trifida \\
\hline Hovenia acerba \\
\hline Ilex asprella \\
\hline Ilex cornuta \\
\hline Ilex hylonoma \\
\hline Ilex pubescens \\
\hline Ilex purpurea \\
\hline Ilex viridis \\
\hline Impatiens furcillata \\
\hline Indigofera ichangensis \\
\hline Indocalamus tessellatus \\
\hline Inula britannica \\
\hline Iris lactea var. chinensis \\
\hline Itea chinensis var. oblonga \\
\hline Ixeris chinensis \\
\hline Ixeris denticulata \\
\hline Ixora chinensis \\
\hline Jasminum lanceolarium \\
\hline Juglans mandshurica \\
\hline Kadsura longipedunculata \\
\hline Kalimeris integrifolia \\
\hline Kalopanax septemlobus \\
\hline Kochia prostrata \\
\hline Kochia scoparia var. sieversiana \\
\hline Kummerowia striata \\
\hline Lamium japonicum \\
\hline Larix olgensis \\
\hline Lasianthus lancilimbus \\
\hline Lathyrus davidii \\
\hline $\begin{array}{l}\text { Lathyrus hedysaroides var. } \\
\text { subsericea }\end{array}$ \\
\hline Laurocerasus spinulosa \\
\hline Laurocerasus zippeliana \\
\hline Leontopodium leontopodioides \\
\hline Leonurus japonicus \\
\hline Lepidium apetalum \\
\hline Lespedeza bicolor \\
\hline Lespedeza davurica \\
\hline Lespedeza formosa \\
\hline Lespedeza hedysaroides \\
\hline Leymus chinensis \\
\hline Ligustrum lucidum \\
\hline Ligustrum sinense \\
\hline Lilium lancifolium \\
\hline
\end{tabular}

NSTEC07

NECT03, NECT04

NECT02, NECT10, NECT15, NECT20, NECT22, NECT23, NECT24, NECT25, NECT26, NECT27

NECT12

NECT10

NECT30, NECT31, NECT32, NECT33

NSTEC11

NSTEC08

NSTEC03

NSTEC10

NSTEC06, NSTEC07, NSTEC08

NSTEC04, NSTEC11

NSTEC07

NECT06, NECT09

NSTEC06

NSTEC07

NECT11, NECT12

NECT10, NECT13, NECT22, NECT25, NECT33

NSTEC06, NSTEC07

NECT04

NECT10, NECT12

NSTEC09

NSTEC12

NECT09, NSTEC14

NSTEC06, NSTEC11, NSTEC12

NECT11

NSTEC03, NSTEC12

NECT23, NECT24, NECT27, NECT29

NECT12, NECT13

NECT10, NECT12

NECT11

NECT05, NECT07

NSTEC06, NSTEC07

NECT04

NECT15

NSTEC06

NSTEC11

NECT15

NECT20

NECT13

NECT03, NECT05, NECT06, NECT07, NECT08, NSTEC13, NSTEC14

NECT01, NECT02, NECT10, NECT15, NECT17, NECT18, NECT19, NECT20, NECT21

NSTEC02

NECT15, NECT20

NECT01, NECT11, NECT12, NECT13, NECT17, NECT18, NECT20, NECT21, NECT22, NECT24, NECT25, NECT26

NSTEC03

NSTEC11

NECT08, NECT09 


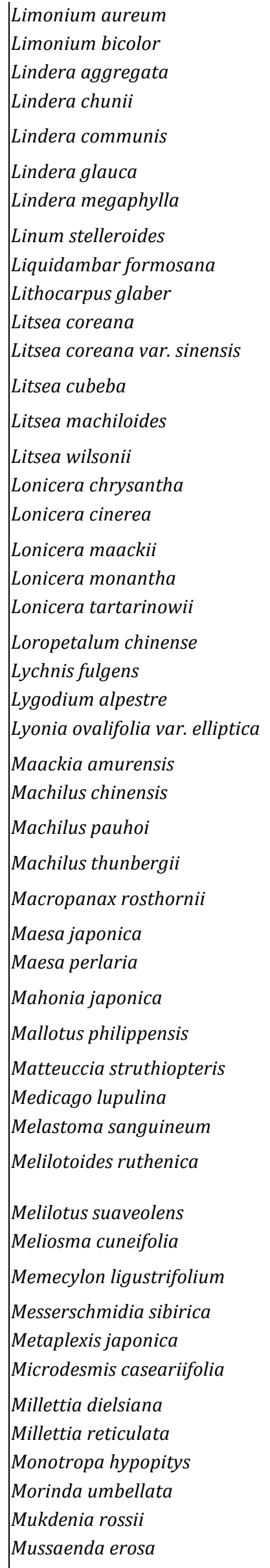

NECT32

NECT13, NECT20, NECT26

NSTEC04, NSTEC06

NSTEC09

NSTEC11

NSTEC03, NSTEC06

NSTEC11

NECT10

NSTEC03, NSTEC05, NSTEC06

NSTEC05, NSTEC06, NSTEC07, NSTEC08, NSTEC11

NSTEC11, NSTEC12

NSTEC10

NSTEC08

NSTEC08

NSTEC06, NSTEC07, NSTEC08

NECT03, NECT05

NSTEC06

NECT06, NECT09, NSTEC14

NECT09

NSTEC13

NSTEC04, NSTEC05, NSTEC06, NSTEC07, NSTEC10, NSTEC12

NECT06, NECT09

NECT09

NSTEC07

NECT05, NECT06

NSTEC09

NSTEC11

NSTEC05

NSTEC11

NSTEC06, NSTEC11, NSTEC12

NSTEC11

NSTEC12

NSTEC11

NECT09

NECT16

NSTEC09

NECT02, NECT10, NECT17, NECT18, NECT20, NECT21, NECT22, NECT24, NECT25, NECT26, NECT27

NECT11

NSTEC09

NSTEC09

NECT13

NECT13

NSTEC09

NSTEC07, NSTEC10, NSTEC11

NSTEC06, NSTEC10

NECT08

NSTEC05, NSTEC06

NECT05

NSTEC08 


\begin{tabular}{|c|c|}
\hline Mussaenda esquirolii & NSTEC12 \\
\hline Mussaenda pubescens & NSTEC07 \\
\hline Myrica rubra & NSTEC05 \\
\hline Neolitsea aurata var zhejinagensis & NSTEC05 \\
\hline Onoclea sensibilis & NECT05 \\
\hline Oreocnide frutescens & NSTEC06 \\
\hline Ormosia glaberrima & NSTEC09 \\
\hline Ormosia henryi & NSTEC05 \\
\hline Orostachys malacophylla & NECT23 \\
\hline Osmanthus cooperi & NSTEC04 \\
\hline Osmanthus fragrans & NSTEC03, NSTEC11 \\
\hline Oxytropis myriophylla & NECT25 \\
\hline Oxytropis sp. & NECT02, NECT25 \\
\hline Paederia scandens & NSTEC03 \\
\hline Paeonia japonica & NECT06, NECT07 \\
\hline Paris verticillata & NECT08 \\
\hline Parthenocissus laetevirens & NSTEC11 \\
\hline Parthenocissus tricuspidata & NSTEC03 \\
\hline Patrinia rupestris & NECT15 \\
\hline Paulownia kawakamii & NSTEC07 \\
\hline Peganum harmala & NECT28, NECT33 \\
\hline Pennisetum flaccidum & NECT15, NECT16, NECT17, NECT18, NECT20 \\
\hline Pericampylus glaucus & NSTEC06, NSTEC07 \\
\hline Periploca sepium & NSTEC13 \\
\hline Peucedanum terebinthaceum & NECT07 \\
\hline Phellodendron amurense & NECT05, NECT06 \\
\hline Philadelphus tenuifolius & NECT03, NECT07, NECT08, NECT09 \\
\hline Phlomis maximowiczii & NECT04, NECT06, NECT09 \\
\hline Phlomis tuberosa & NECT25 \\
\hline Phoebe hunanensis & NSTEC07 \\
\hline Phoebe sheareri & NSTEC11 \\
\hline Photinia beauverdiana & NSTEC11 \\
\hline Photinia davidsoniae & NSTEC12 \\
\hline Photinia glabra & NSTEC04, NSTEC08 \\
\hline Photinia parvifolia & NSTEC07 \\
\hline Photinia serrulata & NSTEC07 \\
\hline Phragmites australis & NECT05, NECT11, NECT13 \\
\hline Phragmites hirsutus & NECT11 \\
\hline Phyllostachys heteroclada & NSTEC03 \\
\hline Picea sp. & NECT07 \\
\hline Picrasma chinensis & NSTEC09 \\
\hline Pinus koraiensis & NECT06, NECT08, NECT09 \\
\hline Pinus massoniana & NSTEC03, NSTEC06, NSTEC10, NSTEC12 \\
\hline Pinus tabuliformis & NECT04, NECT10, NSTEC01, NSTEC02, NSTEC13 \\
\hline Piper chinense & NSTEC09 \\
\hline Piper hancei & NSTEC11 \\
\hline Piper wallichii & NSTEC11 \\
\hline Pistacia chinensis & NSTEC03, NSTEC12, NSTEC13 \\
\hline Pittosporum sahnianum & NSTEC06, NSTEC12 \\
\hline
\end{tabular}




\begin{tabular}{|c|c|}
\hline Pittosporum tobira & NSTEC03 \\
\hline Plantago depressa & NECT12 \\
\hline Platycladus orientalis & NSTEC02, NSTEC13 \\
\hline Platycodon grandiflorus & NECT10, NSTEC02 \\
\hline Pleioblastus amarus & NSTEC05, NSTEC06, NSTEC07 \\
\hline Poa angustifolia & NECT24 \\
\hline Podocarpium podocarpum & NSTEC12 \\
\hline Podocarpium podocarpum var. fallax & NSTEC11 \\
\hline $\begin{array}{l}\text { Podocarpium podocarpum var. } \\
\text { oxyphyllum }\end{array}$ & NSTEC03, NSTEC11 \\
\hline Polygala tenuifolia & NECT01, NECT10 \\
\hline Polygonatum oderatum & NECT04, NECT06, NECT07 \\
\hline Polygonum divaricatum & NECT17, NECT18, NECT20, NECT21, NECT23 \\
\hline Polygonum sibiricum & NECT11, NECT13 \\
\hline Populus davidiana & NECT03, NECT09, NECT10 \\
\hline Potentilla acaulis & NECT21, NECT22, NECT24, NECT24 \\
\hline Potentilla bifurca & NECT21, NECT22, NECT24 \\
\hline Potentilla chinensis & NECT10, NECT12, NECT17, NECT20, NECT21, NECT24, NECT25, NECT26 \\
\hline Potentilla conferta & NECT18 \\
\hline Potentilla discolor & NECT01 \\
\hline Potentilla flagellaris & NECT12 \\
\hline Potentilla tanacetifolia & NECT02 \\
\hline Premna fordii & NSTEC06 \\
\hline Prunus/Malus sp. & NECT06 \\
\hline Prunus padus & NECT04 \\
\hline Psammochloa villosa & NECT24 \\
\hline Psychotria serpens & NSTEC09 \\
\hline Pteridium aquilinum & NECT03, NECT04, NECT05, NECT06, NECT08, NECT09 \\
\hline Puccinella chinampoensis & NECT11 \\
\hline Pueraria lobata & NSTEC10 \\
\hline Pyrus betulifolia & NSTEC13 \\
\hline Quercus acutissima & NSTEC01, NSTEC02, NSTEC03 \\
\hline Quercus aliena & NSTEC03, NSTEC12, NSTEC13 \\
\hline Quercus baronii & NSTEC13 \\
\hline Quercus chenii & NSTEC13 \\
\hline Quercus fabrei & NSTEC01 \\
\hline Quercus mongolica & NECT04, NECT05, NECT06, NECT07, NECT08, NECT09, NECT10, NSTEC14 \\
\hline Quercus serrata var. breviopetiolata & NSTEC02 \\
\hline Radermachera sinica & NSTEC10 \\
\hline Randia cochinchinensis & NSTEC07, NSTEC08, NSTEC09 \\
\hline Ranunculus chinensis & NECT07 \\
\hline Rapanea kwangsiensis & NSTEC10 \\
\hline Raphiolepis indica & NSTEC04 \\
\hline Raphiolepis lanceolata & NSTEC08 \\
\hline Reaumuria soongarica & NECT32 \\
\hline Rhamnus leptophylla & NSTEC12, NSTEC13, NSTEC14 \\
\hline Rhamnus schneideri & NECT04, NECT07 \\
\hline Rhamnus sp. & NECT05, NECT06 \\
\hline Rhaponticum uniflorum & NECT15, NECT25, NECT26, NECT27 \\
\hline Rhododendron mariae & NSTEC08 \\
\hline Rhododendron mariesii & NSTEC04 \\
\hline
\end{tabular}




\begin{tabular}{|c|c|}
\hline Rhododendron ovatum & NSTEC04, NSTEC05 \\
\hline Rhododendron $s p$ & NECT07 \\
\hline Rhodomyrtus tomentosa & NSTEC08 \\
\hline Rhus chinensis & NSTEC13 \\
\hline Ribes amurensis & NECT03 \\
\hline Robinia pseudoacacia & NSTEC01 \\
\hline Rosa cymosa & NSTEC03 \\
\hline Rosa laevigata & NSTEC06 \\
\hline Rosa sp. & NECT03, NECT08, NECT10 \\
\hline Rourea minor & NSTEC09 \\
\hline Rubia cordifolia & NECT09 \\
\hline Rubia sp. & NECT06, NECT08 \\
\hline Rubia sylvatica & NECT04, NECT05, NECT09 \\
\hline Rubus columellaris & NSTEC07 \\
\hline Rubus corchorifolius & NSTEC06, NSTEC07 \\
\hline Rubus ichangensis & NSTEC11 \\
\hline Rubus irenaeus & NSTEC11 \\
\hline Rubus malifolius & NSTEC11 \\
\hline Rubus parvifolius & NSTEC03 \\
\hline Rubus sp. & NECT08 \\
\hline Rubus xanthocarpus & NSTEC14 \\
\hline Sabia swinhoei & NSTEC06, NSTEC11 \\
\hline Sabina chinensis & NSTEC03 \\
\hline Sageretia henryi & NSTEC11, NSTEC12 \\
\hline Sageretia thea & NSTEC06, NSTEC13 \\
\hline Salix gordejevii & NECT16 \\
\hline Salix gracilistyla & NECT05 \\
\hline Salix viminalis & NECT05 \\
\hline Salsola collina & $\begin{array}{l}\text { NECT13, NECT15, NECT18, NECT19, NECT20, NECT22, NECT27, NECT28, } \\
\text { NECT29, NECT30, NECT31, NECT33 }\end{array}$ \\
\hline Salsola passerina & NECT32 \\
\hline Salsola ruthenica & NECT16 \\
\hline Sambucus sp. & NECT08 \\
\hline Sanguisorba officinalis & NECT11 \\
\hline Sanicula chinenesis & NECT09 \\
\hline Sapium discolor & NSTEC08 \\
\hline Sapium rotundifolium & NSTEC10 \\
\hline Sarcandra glabra & NSTEC07, NSTEC09 \\
\hline Sarcosperma arboreum & NSTEC09 \\
\hline Saussurea japonica & NECT02 \\
\hline Saussurea parviflora & NECT02 \\
\hline Schefflera octophylla & NSTEC08 \\
\hline Schima remotiserrata & NSTEC08 \\
\hline Schima superba & NSTEC04, NSTEC05, NSTEC09 \\
\hline Schizonepeta multifida & NECT23 \\
\hline Scorzonera divaricata & NECT28, NECT29, NECT30, NECT31, NECT32 \\
\hline Scorzonera muriculata & NECT32 \\
\hline Scutellaria baicalensis & NECT01, NECT02, NECT18 \\
\hline Scutellaria scordifolia & NECT02 \\
\hline Scutellaria sp. & NECT07 \\
\hline
\end{tabular}

NSTEC04, NSTEC05

NECT07

NSTEC08

NSTEC03

NSTEC06

03, NECT08, NECT10

NECT04, NECT05, NECT09

EC07

STEC06, NSTEC07

NSTEC11

NSTEC03

STEC11

STEC03

NSTEC11, NSTEC12

STEC06, NSTEC13

NECT16

NECT05

NECT13, NECT15, NECT18, NECT19, NECT20, NECT22, NECT27, NECT28, NECT29, NECT30, NECT31, NECT33

NECT32

NECT16

NECT08

NECT11

NECT09

NSTEC08

NSTEC09

NSTEC09

NSTEC08

NECT23

NECT01, NECT02, NECT18

NECT07 


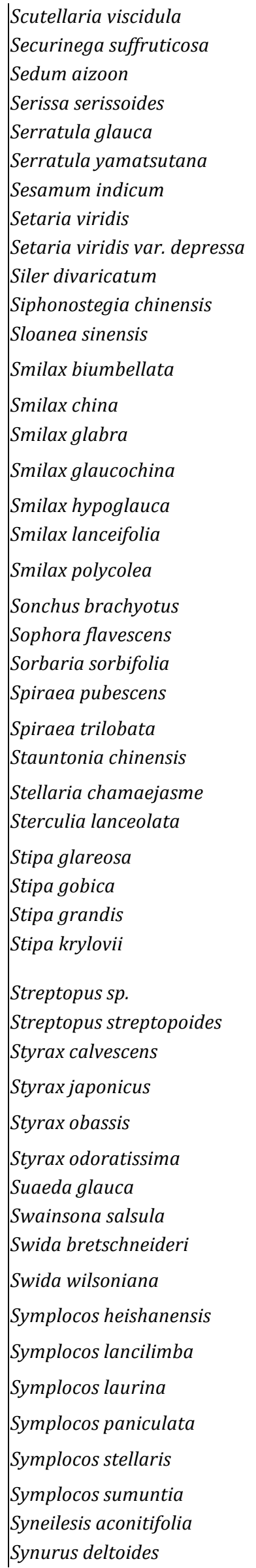

NECT19

NECT04, NECT10

NECT07, NECT09

NSTEC03, NSTEC11, NSTEC12

NECT18

NECT02, NECT15, NECT21, NECT23, NECT25, NECT26

NECT02

NECT10, NECT13, NECT14, NECT15, NECT16, NECT19, NECT26, NECT33

NECT30, NECT31

NECT18, NECT26

NECT10, NECT10

NSTEC07

NSTEC10

NSTEC04, NSTEC05, NSTEC08

NSTEC06

NSTEC03

NSTEC09, NSTEC13

NSTEC07

NSTEC11

NECT10

NECT18

NECT05, NECT06

NSTEC14

NECT23, NSTEC01

NSTEC05

NECT02, NECT23

NSTEC09

NECT29

NECT30, NECT31, NECT32, NECT33

NECT21

NECT01, NECT02, NECT15, NECT22, NECT23, NECT24, NECT25, NECT26, NECT27, NECT28

NECT09

NECT03

NSTEC07

NSTEC05

NSTEC06

NSTEC07, NSTEC10

NECT11, NECT13

NECT13

NSTEC14

NSTEC12

NSTEC05

NSTEC05

NSTEC11

NSTEC03

NSTEC05

NSTEC04, NSTEC05, NSTEC07

NECT04, NECT07

NECT07 


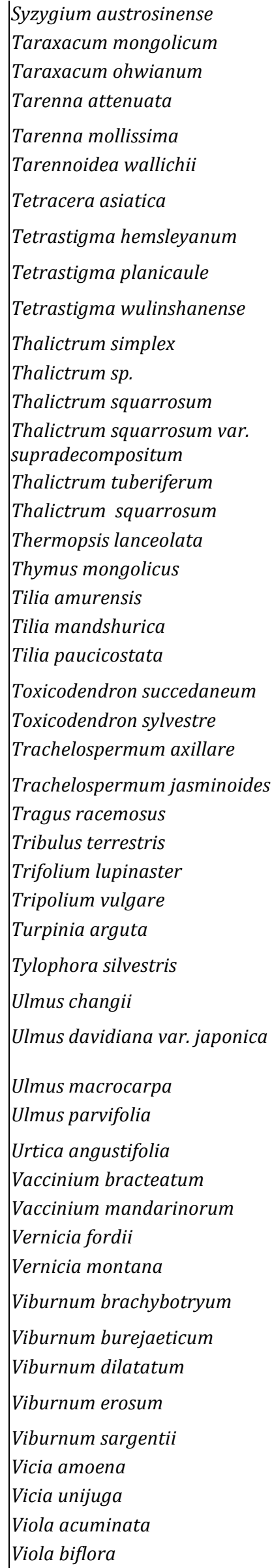

NSTEC07, NSTEC08

NECT02

NECT12, NECT13

NSTEC08

NSTEC06, NSTEC07

NSTEC07

NSTEC09

NSTEC09

NSTEC09

NSTEC11

NECT11

NECT01, NECT08, NECT09, NECT10, NECT26

NECT15, NECT16, NECT18, NECT21

NECT02, NECT 25

NECT03, NECT04, NECT05, NECT06

NECT26

NECT13, NECT23

NECT01, NECT02

NECT03, NECT05, NECT07

NECT03, NECT06, NECT07, NECT08, NECT09

NSTEC14

NSTEC06, NSTEC07, NSTEC08

NSTEC11, NSTEC12

NSTEC06

NSTEC03, NSTEC05, NSTEC10, NSTEC11

NECT02, NECT14, NECT16

NECT02, NECT14, NECT16, NECT19, NECT30, NECT33

NECT07

NECT10

NSTEC11

NSTEC05

NSTEC06

NECT03, NECT04, NECT05, NECT06, NECT09, NECT10, NECT17, NECT21, NSTEC14

NECT08

NSTEC03

NECT03, NECT04

NSTEC06, NSTEC07

NSTEC04, NSTEC05, NSTEC06

NSTEC03, NSTEC06, NSTEC13

NSTEC07

NSTEC11

NECT09

NSTEC11

NSTEC05

NECT05, NECT06, NECT09

NECT04

NECT05, NECT06, NECT07, NECT08

NECT07, NECT08, NECT09

NECT09 
Viola sp.

Viola variegata

Vitex negundo

Vitex negundo var. heterophylla

Vitis amurensis

Vitis bryoniifolia

Vitis heyneana ssp. ficifolia

Wisteria sinensis

Xanthium strumarium

Zanthoxylum echinocarpum

Zanthoxylum podocarpum

Zanthoxylum schinifolium

Zelkova serrata

unidentified chenopod

unidentified forb

unidentified geophyte

unidentified grass

unidentified ground creeper

unidentified Scrophulariaceae

unidentified semi-rosette forb
NECT03, NECT07, NECT08, NECT10

NECT04

NSTEC02, NSTEC03, NSTEC10, NSTEC13

NSTEC01

NECT06, NECT08

NSTEC13

NSTEC01

NSTEC04

NECT12

NSTEC12

NSTEC10

NSTEC02

NSTEC13

NECT27

NECT01, NECT06, NECT08, NECT10

NECT02

NECT01, NECT02, NECT15, NECT17, NECT21

NECT09

NECT02

NECT02 
Table S3: Principal components of climate data based on the country-wide, 10-km grid. Each component (axis) represents a linear combination of variables. Loadings for variables represent the contribution of each variable to the axis. Values are shown in bold when their magnitude $>0.5$.

\begin{tabular}{lrrr} 
& PC 1 & PC 2 & PC 3 \\
\hline MAT & $\mathbf{0 . 8 8 3}$ & -0.422 & 0.179 \\
GDD $_{0}$ & $\mathbf{0 . 8 7 9}$ & -0.411 & 0.142 \\
MTCO & $\mathbf{0 . 8 6 5}$ & -0.144 & 0.166 \\
MTWA & $\mathbf{0 . 6 6 9}$ & $-\mathbf{0 . 6 0 3}$ & 0.120 \\
PAR $_{0}$ & $\mathbf{0 . 6 7 2}$ & $-\mathbf{0 . 6 5 0}$ & 0.234 \\
MAP & $\mathbf{0 . 9 0 3}$ & 0.407 & 0.046 \\
$\alpha$ & $\mathbf{0 . 5 7 8}$ & $\mathbf{0 . 7 4 4}$ & 0.166 \\
MI & $\mathbf{0 . 7 6 8}$ & $\mathbf{0 . 6 2 3}$ & 0.030 \\
PDJF $_{\text {PJJA }}$ & $\mathbf{0 . 8 7 6}$ & 0.166 & -0.387 \\
Timing & $\mathbf{0 . 7 5 9}$ & $\mathbf{0 . 5 4 0}$ & 0.330 \\
Seasonality & -0.639 & 0.100 & $\mathbf{0 . 7 0 2}$ \\
Variance explained & $60.5 \%$ & $21.7 \%$ & $9.0 \%$ \\
\hline
\end{tabular}

